\title{
ON THE ORDER OF GROWTH OF CONVERGENT SERIES OF INDEPENDENT RANDOM VARIABLES
}

\author{
EUNWOO NAM
}

Received 8 May 2003 and in revised form 30 January 2004

For independent random variables, the order of growth of the convergent series $S_{n}$ is studied in this paper. More specifically, if the series $S_{n}$ converges almost surely to a random variable, the tail series is a well-defined sequence of random variables and converges to 0 almost surely. For the almost surely convergent series $S_{n}$, a tail series strong law of large numbers (SLLN) is constructed by investigating the duality between the limiting behavior of partial sums and that of tail series.

\section{Introduction}

Let $\left\{X_{n}, n \geq 1\right\}$ be a sequence of random variables defined on a probability space $(\Omega, \mathscr{F}$, $P)$ and, as usual, their partial sums are denoted by $S_{n}=\sum_{j=1}^{n} X_{j}, n \geq 1$. The sequence of random variables $\left\{X_{n}, n \geq 1\right\}$ (such that the series $S_{n}$ diverges almost surely (a.s.)) is said to obey the strong law of large numbers (SLLN) with positive norming constants $\left\{a_{n}, n \geq 1\right\}$ if

$$
\frac{S_{n}}{a_{n}} \longrightarrow 0 \quad \text { a.s. }
$$

If the series $S_{n}$ converges a.s. to a random variable $S$, then (set $S_{0}=X_{0}=0$ ) the tail series

$$
T_{n}=S-S_{n-1}=\sum_{j=n}^{\infty} X_{j}, \quad n \geq 1,
$$

is a well-defined sequence of random variables and converges to 0 a.s. In the same way, a sequence of random variables $\left\{X_{n}, n \geq 1\right\}$ is said to obey the tail series SLLN with norming constants $\left\{b_{n}, n \geq 1\right\}$ if the tail series $T_{n}$ is well defined and for a given sequence of positive constants with $b_{n}=o(1)$,

$$
\frac{T_{n}}{b_{n}} \longrightarrow 0 \quad \text { a.s. }
$$


In this paper, for independent random variables, we will be concerned with the rate in which $S_{n}$ converges to a random variable $S$, or equivalently, in which the tail series $T_{n}$ converges to 0 . As will be seen, many results for partial sums $S_{n}$ can be paired with analogous results for tail series $T_{n}$. Most of the limit laws for tail series have been developed by discovering and investigating this duality.

Pioneering work on the limiting behavior of $\left\{T_{n}, n \geq 1\right\}$ was conducted by Chow and Teicher [3], where they obtained for a tail series of suitably bounded and independent summands a counterpart to Kolmogorov's celebrated law of the iterated logarithm (LIL) (see, e.g., Chow and Teicher [4, Theorem 10.2.1, page 373] or Petrov [15, Theorem 7.1, page 239]). Barbour [1] then established a tail series analogue of the Lindeberg-Feller version of the central limit theorem. Other numerous investigations on the tail series LIL problem have followed; see Heyde [7], Wellner [20], Kesten [8], Budianu [2], Chow et al. [5], Klesov [9], Rosalsky [16], Mikosch [11], and Tomkins [19] for such work. The tail series SLLN problem was studied by Klesov [9, 10], Mikosch [11], and Nam and Rosalsky [12]. Also, Nam and Rosalsky [13] constructed a limit law which implies a tail series weak law of large numbers, and then this result was not only generalized by Sung and Volodin [18], but it was also generalized and simplified by Rosalsky and Rosenblatt [17].

For independent random variables, Petrov [14] proved a SLLN for partial sums, and Klesov $[9,10]$ then developed a tail series SLLN which provided a tail series counterpart to Petrov's [14] SLLN. It will be shown that Klesov's [10] tail series SLLN can be extended to a wider class of random variables in this paper. As will become apparent, the formulation and proof of the ensuing Theorem 3.1 owe much to the work of Klesov [9, 10].

\section{Preliminary lemmas}

Several lemmas are needed to establish the main results. Some of Klesov's [9, 10] work will now be described. Let $\Psi^{*}$ be the class of positive and nondecreasing functions $\psi^{*}(x)$ such that the series $\sum_{n=1}^{\infty} 1 / n \psi^{*}(n)$ converges and $x \psi^{*}\left(x^{-1}\right)$ tends monotonically to 0 as $x \downarrow 0$.

LEMma 2.1 (Klesov $[9,10])$. Let $\left\{c_{n}, n \geq 1\right\}$ be a sequence of nonnegative constants such that $\sum_{n=1}^{\infty} c_{n}<\infty$. If $C_{n} \equiv \sum_{j=n}^{\infty} c_{j}>0, n \geq 1$, then $\sum_{n=1}^{\infty}\left(c_{n} / C_{n} \psi^{*}\left(C_{n}^{-1}\right)\right)<\infty$ obtains for each function $\psi^{*}(x) \in \Psi^{*}$.

Lemma 2.2 (Heyde [7], Rosalsky [16], and Klesov [10]). Let $\left\{x_{n}, n \geq 1\right\}$ be a sequence of constants and let $\left\{b_{n}, n \geq 1\right\}$ be a sequence of positive constants with $b_{n} \downarrow 0$. If the series $\sum_{n=1}^{\infty}\left(x_{n} / b_{n}\right)$ converges, then $\left(1 / b_{n}\right) \sum_{j=n}^{\infty} x_{j} \longrightarrow 0$.

Lemma 2.3 (Petrov [15, Theorem 6.4, page 207]). Let $\left\{X_{n}, n \geq 1\right\}$ be independent random variables and let $\left\{g_{n}(x), n \geq 1\right\}$ be nondecreasing functions defined on $[0, \infty)$ such that

$$
g_{n}(0)=0, \quad \lim _{x \rightarrow \infty} g_{n}(x)=\infty, \quad n \geq 1
$$

Suppose that one of the following three conditions prevails:

(i) $x / g_{n}(x)$ is nondecreasing in $x>0$ for each $n \geq 1$;

(ii) $g_{n}(x) / x$ and $x^{2} / g_{n}(x)$ are nondecreasing in $x>0$, and also $E\left(X_{n}\right)=0$, for each $n \geq 1$; 
(iii) $x^{2} / g_{n}(x)$ is nondecreasing in $x>0$, and $X_{n}$ has a symmetric distribution, for each $n \geq 1$.

Further, let $\left\{b_{n}, n \geq 1\right\}$ be a sequence of positive constants. If the series

$$
\sum_{n=1}^{\infty} \frac{E\left(g_{n}\left(\left|X_{n}\right|\right)\right)}{g_{n}\left(b_{n}\right)}<\infty
$$

then the series $\sum_{n=1}^{\infty} b_{n}^{-1} X_{n}$ converges a.s.

Under hypothesis (i) or (ii), Lemma 2.3 was proved, for the case $g_{n} \equiv g$, by Chung [6]. Using Lemmas 2.2 and 2.3, we obtain the following lemma. Not only does Lemma 2.4 imply Klesov [10, Assertion 3], but it also obtains a tail series analogue of Petrov [15, Theorem 6.5, page 209]. Note that the assumptions of the lemma entail (3.2) which, as will be demonstrated in the proof of Theorem 3.1, ensures that $\left\{T_{n}, n \geq 1\right\}$ is a welldefined sequence of random variables.

Lemma 2.4. Let $\left\{X_{n}, n \geq 1\right\}$ be independent random variables and let $\left\{g_{n}(x), n \geq 1\right\}$ be nondecreasing functions defined on $[0, \infty)$ satisfying (2.1). Suppose that one of conditions $(i)$, (ii), and (iii) of Lemma 2.3 holds. Let $\left\{b_{n}, n \geq 1\right\}$ be a sequence of positive constants with $b_{n} \downarrow 0$. If $g_{n}\left(b_{n}\right)=O(1)$ and (2.2) holds, then the tail series SLLN $T_{n} / b_{n} \rightarrow 0$ a.s. obtains.

\section{The main result}

For independent random variables $\left\{X_{n}, n \geq 1\right\}$, a tail series SLLN, which implies the result of Klesov [10, Assertion 4] by taking $g_{n} \equiv g$ under hypothesis (i) or (ii), is obtained in Theorem 3.1. Assume that $\left\{X_{n}, n \geq 1\right\}$ are not eventually degenerate at 0 .

Theorem 3.1. Let $\left\{X_{n}, n \geq 1\right\}$ be independent random variables and let $\left\{g_{n}(x), n \geq 1\right\}$ be strictly increasing functions defined on $[0, \infty)$ such that (2.1) holds, and assume that

$$
g_{n}(x) \text { is nondecreasing in } n \text { for each fixed } x>0 .
$$

Suppose that one of the following three conditions prevails:

(i) $x / g_{n}(x)$ is nondecreasing in $x>0$ for each $n \geq 1$;

(ii) $g_{n}(x) / x$ and $x^{2} / g_{n}(x)$ are nondecreasing in $x>0$; and also $E\left(X_{n}\right)=0$, for each $n \geq 1$;

(iii) $x^{2} / g_{n}(x)$ is nondecreasing in $x>0$, and $X_{n}$ has a symmetric distibution, for each $n \geq 1$.

If the series

$$
\sum_{n=1}^{\infty} E\left(g_{n}\left(\left|X_{n}\right|\right)\right)<\infty
$$

then setting $B_{n}=\sum_{j=n}^{\infty} E\left(g_{j}\left(\left|X_{j}\right|\right)\right), n \geq 1$, the tail series $S L L N$

$$
\frac{T_{n}}{g_{n}^{-1}\left(B_{n} \psi^{*}\left(B_{n}^{-1}\right)\right)} \longrightarrow 0 \quad \text { a.s. for each function } \psi^{*}(x) \in \Psi^{*},
$$

obtains, where $g_{n}^{-1}$ denotes the inverse function of $g_{n}$. 
Remark 3.2. Given that $g_{n}(x)$ is a nondecreasing function, each $g_{n}(x)$ is necessarily a continuous function for each of conditions (i), (ii), and (iii).

Proof of Theorem 3.1. First, it will be verified, by employing the Kolmogorov three-series criterion, that $\sum_{n=1}^{\infty} X_{n}$ converges a.s. For $n \geq 1$,

$$
P\left\{\left|X_{n}\right|>1\right\}=P\left\{g_{n}\left(\left|X_{n}\right|\right)>g_{n}(1)\right\} \leq \frac{E\left(g_{n}\left(\left|X_{n}\right|\right)\right)}{g_{n}(1)}
$$

by the Markov inequality, and so $\sum_{n=1}^{\infty} P\left\{\left|X_{n}\right|>1\right\}<\infty$. Under hypothesis (i), note that $x^{2} \leq g_{n}{ }^{2}(x) / g_{n}{ }^{2}(1) \leq g_{n}(x) / g_{1}(1), x \leq 1$. Now, under hypothesis (ii) or (iii), also observe that $x^{2} / g_{n}(x) \leq 1 / g_{n}(1)$ and so $x^{2} \leq g_{n}(x) / g_{1}(1), x \leq 1$. Thus

$$
E\left(X_{n}^{2} I_{\left[\left|X_{n}\right| \leq 1\right]}\right) \leq \frac{E\left(g_{n}\left(\left|X_{n}\right| I_{\left[\left|X_{n}\right| \leq 1\right]}\right)\right)}{g_{1}(1)} \leq \frac{E\left(g_{n}\left(\left|X_{n}\right|\right)\right)}{g_{1}(1)}
$$

implying that $\sum_{n=1}^{\infty} \operatorname{Var}\left(X_{n} I_{\left[\left|X_{n}\right| \leq 1\right]}\right)<\infty$ for each case. Furthermore, under hypothesis (i), since $x / g_{n}(x) \leq 1 / g_{n}(1), x \leq 1$,

$$
E\left(X_{n} I_{\left[\left|X_{n}\right| \leq 1\right]}\right) \leq \frac{E\left(g_{n}\left(\left|X_{n}\right| I_{\left[\left|X_{n}\right| \leq 1\right]}\right)\right)}{g_{n}(1)} \leq \frac{E\left(g_{n}\left(\left|X_{n}\right|\right)\right)}{g_{1}(1)} .
$$

Also under hypothesis (ii), since $x / g_{n}(x) \leq 1 / g_{n}(1), x>1$,

$$
\left|E\left(X_{n} I_{\left[\left|X_{n}\right| \leq 1\right]}\right)\right|=\left|E\left(X_{n} I_{\left[\left|X_{n}\right|>1\right]}\right)\right| \leq \frac{E\left(g_{n}\left(\left|X_{n}\right| I_{\left[\left|X_{n}\right|>1\right]}\right)\right)}{g_{n}(1)} .
$$

In the case of (iii), note that $E\left(X_{n} I_{\left[\left|X_{n}\right| \leq 1\right]}\right)=0$. Then $\sum_{n=1}^{\infty} E\left(X_{n} I_{\left[\left|X_{n}\right| \leq 1\right]}\right)<\infty$ is ensured for each case by (3.2). Therefore, the Kolmogorov three-series criterion guarantees that $\left\{T_{n}, n \geq 1\right\}$ is a well-defined sequence of random variables.

Next, let $c_{n}=E\left(g_{n}\left(\left|X_{n}\right|\right)\right), n \geq 1$, and note that $B_{n}=\sum_{j=n}^{\infty} c_{j}>0$; then Lemma 2.1 ensures that for each function $\psi^{*}(x) \in \Psi^{*}$,

$$
\sum_{n=1}^{\infty} \frac{E\left(g_{n}\left(\left|X_{n}\right|\right)\right)}{g_{n}\left(g_{n}^{-1}\left(B_{n} \psi^{*}\left(B_{n}^{-1}\right)\right)\right)}=\sum_{n=1}^{\infty} \frac{E\left(g_{n}\left(\left|X_{n}\right|\right)\right)}{B_{n} \psi^{*}\left(B_{n}^{-1}\right)}<\infty
$$

Therefore, by setting $b_{n}=g_{n}^{-1}\left(B_{n} \psi^{*}\left(B_{n}^{-1}\right)\right)$, the theorem is obtained by Lemma 2.4.

The counterpart of Theorem 3.1 for partial sums, which generalizes Petrov [14, Theorem 5 or 15, Theorem 6.13, page 220], can be proved by the same argument as in Theorem 3.1. In the following corollaries, we obtain two truncated versions of Theorem 3.1, which improve the result of the theorem.

Corollary 3.3. Let $\left\{X_{n}, n \geq 1\right\}$ be independent random variables and let $\left\{g_{n}(x), n \geq 1\right\}$ be strictly increasing functions defined on $[0, \infty)$ satisfying (2.1) and (3.1). Suppose that one 
of conditions (i), (ii), and (iii) of Theorem 3.1 holds. If

$$
\begin{gathered}
\sum_{n=1}^{\infty} P\left\{\left|X_{n}\right|>C_{n}\right\}<\infty, \\
\sum_{n=1}^{\infty} E\left(g_{n}\left(\left|X_{n} I_{\left[\left|X_{n}\right| \leq C_{n}\right]}\right|\right)\right)<\infty
\end{gathered}
$$

are satisfied for some sequence of positive constants $\left\{C_{n}, n \geq 1\right\}$, then, setting $B_{n}^{*}=$ $\sum_{j=n}^{\infty} E\left(g_{j}\left(\left|X_{j} I_{\left[\left|X_{j}\right| \leq C_{j}\right]}\right|\right)\right), n \geq 1$, the tail series SLLN

$$
\frac{T_{n}}{g_{n}^{-1}\left(B_{n}^{*} \psi^{*}\left(B_{n}^{*-1}\right)\right)} \longrightarrow 0 \quad \text { a.s. for each function } \psi^{*}(x) \in \Psi^{*},
$$

obtains, where $g_{n}^{-1}$ denotes the inverse function of $g_{n}$.

Remark 3.4. With the aid of the Borel-Cantelli lemma, a necessary condition for (3.11) to hold is given by (3.9) with $C_{n}=g_{n}^{-1}\left(B_{n}^{*} \psi^{*}\left(B_{n}^{*-1}\right)\right)$.

Proof of Corollary 3.3. Set $Z_{n}=X_{n} I_{\left[\left|X_{n}\right| \leq C_{n}\right]}, n \geq 1$. Then, by applying Theorem 3.1 to the random variables $\left\{Z_{n}, n \geq 1\right\}$, the tail series $T_{n}^{*} \equiv \sum_{j=n}^{\infty} Z_{j}$ is well defined and the tail series SLLN

$$
\frac{T_{n}^{*}}{g_{n}^{-1}\left(B_{n}^{*} \psi^{*}\left(B_{n}^{*-1}\right)\right)} \longrightarrow 0 \text { a.s. for each function } \psi^{*}(x) \in \Psi^{*},
$$

obtains. Since $\left\{X_{n}, n \geq 1\right\}$ and $\left\{X_{n} I_{\left[\left|X_{n}\right| \leq C_{n}\right]}, n \geq 1\right\}$ are equivalent in the sense of Khintchine, $\left\{T_{n}, n \geq 1\right\}$ is well defined and the corollary follows.

Corollary 3.5. Let $\left\{X_{n}, n \geq 1\right\}$ be independent random variables and let $\left\{g_{n}(x), n \geq 1\right\}$ be strictly increasing functions defined on $[0, \infty)$ satisfying (2.1) and (3.1). Suppose that one of conditions (i), (ii), and (iii) of Theorem 3.1 holds. If (3.9) and

$$
\sum_{n=1}^{\infty} E\left(g_{n}\left(\left|X_{n} I_{\left[\left|X_{n}\right| \leq C_{n}\right]}-E\left(X_{n} I_{\left[\left|X_{n}\right| \leq C_{n}\right]}\right)\right|\right)\right)<\infty
$$

are satisfied for some sequence of positive constants $\left\{C_{n}, n \geq 1\right\}$, then, setting $\tilde{B}_{n}=$ $\sum_{j=n}^{\infty} E\left(g_{j}\left(\left|X_{j} I_{\left[\left|X_{j}\right| \leq C_{j}\right]}-E\left(X_{j} I_{\left[\left|X_{j}\right| \leq C_{j}\right]}\right)\right|\right)\right)$ and

$$
\tilde{T}_{n}=\sum_{j=n}^{\infty}\left\{X_{j}-E\left(X_{j} I_{\left[\left|X_{j}\right| \leq C_{j}\right]}\right)\right\}, \quad n \geq 1,
$$

the tail series SLLN

$$
\frac{\tilde{T}_{n}}{g_{n}^{-1}\left(\tilde{B}_{n} \psi^{*}\left(\tilde{B}_{n}^{-1}\right)\right)} \longrightarrow 0 \quad \text { a.s. for each function } \psi^{*}(x) \in \Psi^{*},
$$

obtains, where $g_{n}^{-1}$ denotes the inverse function of $g_{n}$. 
164 The order of growth of convergent series

Proof. Set

$$
Z_{n}=X_{n} I_{\left[\left|X_{n}\right| \leq C_{n}\right]}-E\left(X_{n} I_{\left[\left|X_{n}\right| \leq C_{n}\right]}\right), \quad n \geq 1
$$

Then the corollary follows from (3.9) and (3.13) by the argument in the proof of Corollary 3.3 mutatis mutandis.

\section{Examples}

Three examples are provided to illustrate the current results as well as to compare some of them with related results in the literature.

Example 4.1. Let $\left\{X_{n}, n \geq 1\right\}$ be independent random variables such that

$$
P\left\{X_{n}=\frac{1}{n^{\alpha}}\right\}=P\left\{X_{n}=-\frac{1}{n^{\alpha}}\right\}=\frac{1}{2}, \quad \alpha>\frac{1}{2} .
$$

Theorem 3.1 and the theorem of Klesov [10, Assertion 4] will be employed to determine the rate of almost sure convergence of the series $S_{n}=\sum_{j=1}^{n} X_{j}$. Define

$$
g_{n}(x)=n^{\alpha-1 / 2} x^{2}, \quad n \geq 1,
$$

and $g(x)=g_{1}(x)=x^{2}, x \geq 0$. Then

$$
E\left(g_{n}\left(\left|X_{n}\right|\right)\right)=n^{-(\alpha+1 / 2)}, \quad E\left(g\left(\left|X_{n}\right|\right)\right)=n^{-2 \alpha},
$$

implying that all the hypotheses of Theorem 3.1 as well as Klesov's [10] theorem are satisfied. Now, for $n \geq 1$,

$$
\begin{gathered}
B_{n}=\sum_{j=n}^{\infty} E\left(g_{j}\left(\left|X_{j}\right|\right)\right) \sim M_{1} n^{-(\alpha-1 / 2)}, \\
\mathscr{B}_{n}=\sum_{j=n}^{\infty} E\left(g\left(\left|X_{j}\right|\right)\right) \sim M_{2} n^{-(2 \alpha-1)},
\end{gathered}
$$

where $M_{1}=(\alpha-1 / 2)^{-1}$ and $M_{2}=M_{1} / 2$. If $\psi^{*}(x)$ is taken to be the function

$$
\psi^{*}(x)=\sqrt{x}
$$

then

$$
B_{n} \psi^{*}\left(B_{n}^{-1}\right) \sim M_{1}^{1 / 2} n^{-(1 / 2)(\alpha-1 / 2)}, \quad \mathscr{B}_{n} \psi^{*}\left(\mathscr{B}_{n}^{-1}\right) \sim M_{2}^{1 / 2} n^{-(\alpha-1 / 2)},
$$

and so, respectively,

$$
\begin{gathered}
g_{n}^{-1}\left(B_{n} \psi^{*}\left(B_{n}^{-1}\right)\right) \sim M_{1}^{1 / 4} n^{-(3 / 4)(\alpha-1 / 2)}, \\
g^{-1}\left(\mathscr{S}_{n} \psi^{*}\left(\mathscr{S}_{n}^{-1}\right)\right) \sim M_{2}^{1 / 4} n^{-(1 / 2)(\alpha-1 / 2)} .
\end{gathered}
$$


Thus, by applying Theorem 3.1 and the theorem of Klesov [10], the tail series SLLNs

$$
\begin{array}{ll}
n^{(3 / 4)(\alpha-1 / 2)} T_{n} \longrightarrow 0 & \text { a.s., } \\
n^{(1 / 2)(\alpha-1 / 2)} T_{n} \longrightarrow 0 & \text { a.s., }
\end{array}
$$

obtain, respectively. Hence, recalling $\alpha>1 / 2$, (4.9) dominates (4.10). Therefore, Theorem 3.1 gives us a sharper result than that which can be obtained by Klesov's [10] theorem with $\psi^{*}(x)$ as in (4.6).

Next, by taking

$$
\psi^{*}(x)=\left(\log _{1} x\right)^{1+\varepsilon}, \quad \varepsilon>0,
$$

where

$$
\log _{1} x=\left\{\begin{array}{ll}
\log x & \text { if } x \geq e, \\
e^{-1} x & \text { if } x<e,
\end{array} \text { for the natural logarithm } \log x(x \geq e),\right.
$$

the two relations in (4.4) and (4.5) yield the asymptotic relations

$$
\begin{aligned}
B_{n} \psi^{*}\left(B_{n}^{-1}\right) & \sim M_{3} n^{-(\alpha-1 / 2)}\left(\log _{1} n\right)^{1+\varepsilon}, \\
\mathscr{B}_{n} \psi^{*}\left(\mathscr{B}_{n}^{-1}\right) & \sim M_{4} n^{-(2 \alpha-1)}\left(\log _{1} n\right)^{1+\varepsilon},
\end{aligned}
$$

where $M_{3}=(\alpha-1 / 2)^{\varepsilon}$ and $M_{4}=2^{\varepsilon} M_{3}$, and so, respectively,

$$
\begin{aligned}
g_{n}^{-1}\left(B_{n} \psi^{*}\left(B_{n}^{-1}\right)\right) & \sim M_{3}^{1 / 2} n^{-(\alpha-1 / 2)}\left(\log _{1} n\right)^{(1+\varepsilon) / 2}, \\
g^{-1}\left(\mathscr{B}_{n} \psi^{*}\left(\mathscr{B}_{n}^{-1}\right)\right) & \sim M_{4}^{1 / 2} n^{-(\alpha-1 / 2)}\left(\log _{1} n\right)^{(1+\varepsilon) / 2} .
\end{aligned}
$$

Hence, by either Theorem 3.1 or the theorem of Klesov [10], the tail series SLLN

$$
\frac{n^{\alpha-1 / 2}}{\left(\log _{1} n\right)^{(1+\varepsilon) / 2}} T_{n} \longrightarrow 0 \quad \text { a.s., }
$$

obtains. Therefore, there is no advantage of Theorem 3.1 over Klesov's [10] theorem when $\psi^{*}(x)$ is as in (4.11).

In particular, by taking $\alpha=1$ in Example 4.1, the harmonic series $S_{n}=\sum_{j=1}^{n} X_{j}$ with a random choice of signs is obtained in the following example.

Example 4.2. Let $\left\{X_{n}, n \geq 1\right\}$ be independent random variables such that

$$
P\left\{X_{n}=\frac{1}{n}\right\}=P\left\{X_{n}=-\frac{1}{n}\right\}=\frac{1}{2}, \quad n \geq 1 .
$$

Let $0<\beta<1$ and define

$$
g_{n}(x)=n^{1-\beta} x^{2}, \quad n \geq 1,
$$


and $g(x)=g_{1}(x)=x^{2}, x \geq 0$. Then

$$
E\left(g_{n}\left(\left|X_{n}\right|\right)\right)=n^{-(1+\beta)}, \quad E\left(g\left(\left|X_{n}\right|\right)\right)=n^{-2},
$$

and so, all the hypotheses of both Theorem 3.1 and Klesov's [10] theorem are satisfied. Now, for $n \geq 1$,

$$
B_{n}=\sum_{j=n}^{\infty} E\left(g_{j}\left(\left|X_{j}\right|\right)\right) \sim M n^{-\beta}, \quad \mathscr{B}_{n}=\sum_{j=n}^{\infty} E\left(g\left(\left|X_{j}\right|\right)\right) \sim n^{-1},
$$

where $M=\beta^{-1}$. If $\psi^{*}(x)$ is taken to be the function as in (4.6), then

$$
B_{n} \psi^{*}\left(B_{n}^{-1}\right) \sim M^{1 / 2} n^{-\beta / 2}, \quad \mathscr{B}_{n} \psi^{*}\left(\mathscr{B}_{n}^{-1}\right) \sim n^{-1 / 2},
$$

and so, respectively,

$$
g_{n}^{-1}\left(B_{n} \psi^{*}\left(B_{n}^{-1}\right)\right) \sim M^{1 / 4} n^{-(1 / 2-\beta / 4)}, \quad g^{-1}\left(\mathscr{B}_{n} \psi^{*}\left(\mathscr{B}_{n}^{-1}\right)\right) \sim n^{-1 / 4} .
$$

Thus, by applying Theorem 3.1, we obtain the tail series SLLN

$$
n^{1 / 2-\beta / 4} T_{n} \longrightarrow 0 \quad \text { a.s., }
$$

which dominates the tail series SLLN

$$
n^{1 / 4} T_{n} \longrightarrow 0 \quad \text { a.s. }
$$

given by the theorem of Klesov [10]. Hence, Theorem 3.1 gives us a sharper result than that which can be obtained by Klesov's [10] theorem. Also, if we include $\alpha=1$ in (4.9) for the harmonic series, (4.22) with $\beta<1 / 2$ dominates (4.9). Therefore, by taking $g_{n}(x)$ as in (4.17) instead of as in (4.2), this example gives us a better result than that which was obtained in Example 4.1.

Example 4.3. Let $\left\{X_{n}, n \geq 1\right\}$ be independent random variables such that

$$
P\left\{X_{n}=1\right\}=1-\frac{1}{n^{2}}, \quad P\left\{X_{n}=e^{n}\right\}=\frac{1}{n^{2}}, \quad n \geq 1 .
$$

Let $1 / 2<p \leq 1$ and let $g_{n}(x) \equiv|x|^{p}, n \geq 1$. Then, by setting $C_{n} \equiv 1$ for all $n \geq 1$, all the assumptions of Corollary 3.5 are satisfied since

$$
E\left(g_{n}\left(\left|X_{n} I_{\left[\left|X_{n}\right| \leq C_{n}\right]}-E\left(X_{n} I_{\left[\left|X_{n}\right| \leq C_{n}\right]}\right)\right|\right)\right)=\frac{1}{n^{2 p}}\left(1-\frac{1}{n^{2}}\right)+\left(1-\frac{1}{n^{2}}\right)^{p} \frac{1}{n^{2}} .
$$

Next, for $n \geq 1$,

$$
\tilde{B}_{n}=\sum_{j=n}^{\infty} E\left(g_{n}\left(\left|X_{n} I_{\left[\left|X_{n}\right| \leq C_{n}\right]}-E\left(X_{n} I_{\left[\left|X_{n}\right| \leq C_{n}\right]}\right)\right|\right)\right) \sim M^{-1} n^{-(2 p-1)},
$$


where $M=2 p-1$. If $\psi^{*}(x)$ is taken to be the function as in (4.11), then

$$
\tilde{B}_{n} \psi^{*}\left(\tilde{B}_{n}^{-1}\right) \sim M^{\varepsilon} n^{-(2 p-1)}\left(\log _{1} n\right)^{1+\varepsilon}=o(1), \quad \varepsilon>0,
$$

and so

$$
g_{n}^{-1}\left(\tilde{B}_{n} \psi^{*}\left(\tilde{B}_{n}^{-1}\right)\right) \sim M^{\varepsilon / p} n^{-(2-1 / p)}\left(\log _{1} n\right)^{(1+\varepsilon) / p}
$$

Thus, the tail series SLLN

$$
\frac{n^{2-1 / p}}{\left(\log _{1} n\right)^{(1+\varepsilon) / p}} \tilde{T}_{n} \longrightarrow 0 \quad \text { a.s., }
$$

obtains by Corollary 3.5, where the tail series $\left\{\tilde{T}_{n}, n \geq 1\right\}$ are defined as in (3.14).

\section{Acknowledgment}

The author would like to thank the referee for carefully reading the manuscript and for some remarks which helped to improve the presentation of the paper.

\section{References}

[1] A. D. Barbour, Tail sums of convergent series of independent random variables, Proc. Cambridge Philos. Soc. 75 (1974), 361-364.

[2] G. Budianu, On the law of the iterated logarithm for tail-sums of random variables, Stud. Cerc. Mat. 33 (1981), no. 2, 149-158 (Romanian).

[3] Y. S. Chow and H. Teicher, Iterated logarithm laws for weighted averages, Z. Wahrsch. Verw. Gebiete 26 (1973), 87-94.

[4] _ Probability Theory. Independence, Interchangeability, Martingales, 3rd ed., Springer Texts in Statistics, Springer-Verlag, New York, 1997.

[5] Y. S. Chow, H. Teicher, C. Z. Wei, and K. F. Yu, Iterated logarithm laws with random subsequences, Z. Wahrsch. Verw. Gebiete 57 (1981), no. 2, 235-251.

[6] K.-L. Chung, Note on some strong laws of large numbers, Amer. J. Math. 69 (1947), 189-192.

[7] C. C. Heyde, On central limit and iterated logarithm supplements to the martingale convergence theorem, J. Appl. Probability 14 (1977), no. 4, 758-775.

[8] H. Kesten, The speed of convergence of a martingale, Israel J. Math. 32 (1979), no. 1, 83-96.

[9] O. I. Klesov, The rate of convergence of series of random variables, Ukrain. Mat. Zh. 35 (1983), no. 3, 309-314 (Russian), translated in Ukrainian Math. J. 35 (1983), 264-268.

[10] - Rate of convergence of certain random series, Teor. Imovir. Mat. Stat. (1984), no. 30, 81-92 (Russian), translated in Theory Probab. Math. Statist. (1985), no. 30, 91-101.

[11] T. Mikosch, Almost sure behavior of tail series in functional spaces, Anal. Math. 16 (1990), no. 2, 123-133.

[12] E. Nam and A. Rosalsky, On the almost certain rate of convergence of series of independent random variables, J. Korean Statist. Soc. 24 (1995), no. 1, 91-109.

[13] On the rate of convergence of series of random variables, Teor. Ĭmovīr. Mat. Stat. (1995), no. 52, 120-131 (Ukrainian), translated in Theory Probab. Math. Statist. (1996), no. 52, $129-140$.

[14] V. V. Petrov, The strong law of large numbers, Teor. Verojatnost. i Primenen. 14 (1969), 193-202 (Russian), translated in Theor. Probab. Appl. 14 (1969), 183-192. 


\section{The order of growth of convergent series}

[15] Limit Theorems of Probability Theory. Sequences of Independent Random Variables, Oxford Studies in Probability, vol. 4, The Clarendon Press, Oxford University Press, New York, 1995.

[16] A. Rosalsky, Almost certain limiting behavior of the tail series of independent summands, Bull. Inst. Math. Acad. Sinica 11 (1983), no. 2, 185-208.

[17] A. Rosalsky and J. Rosenblatt, On convergence of series of random variables with applications to martingale convergence and to convergence of series with orthogonal summands, Stochastic Anal. Appl. 16 (1998), no. 3, 553-566.

[18] S. H. Sung and A. I. Volodin, On convergence of series of independent random variables, Bull. Korean Math. Soc. 38 (2001), no. 4, 763-772.

[19] R. J. Tomkins, Rates of convergence for tail series, J. Statist. Plann. Inference 43 (1995), no. 1-2, $77-85$.

[20] J. A. Wellner, A strong invariance theorem for the strong law of large numbers, Ann. Probab. 6 (1978), no. 4, 673-679.

Eunwoo Nam: Department of Mathematical Sciences, United States Air Force Academy (USAFA), CO 80840, USA

E-mail address: eunwoo.nam@usafa.af.mil 


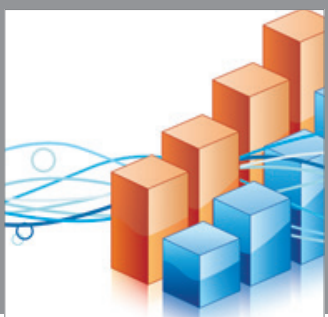

Advances in

Operations Research

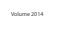

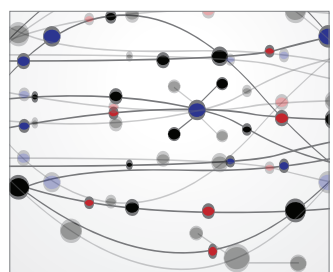

\section{The Scientific} World Journal
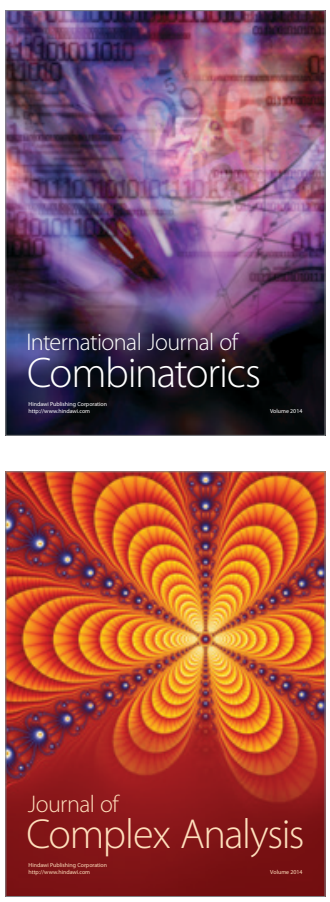

International Journal of

Mathematics and

Mathematical

Sciences
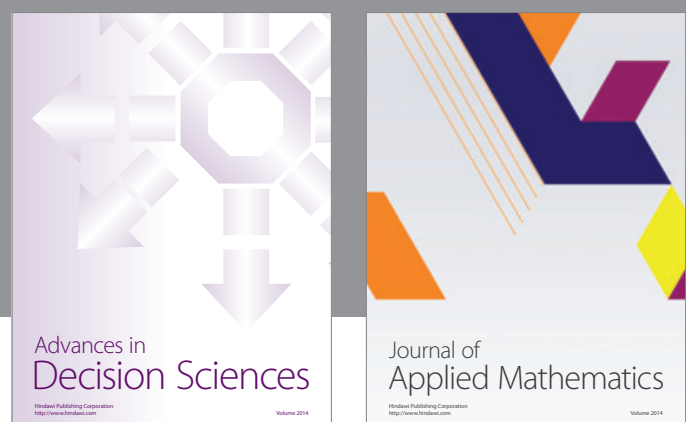

Journal of

Applied Mathematics
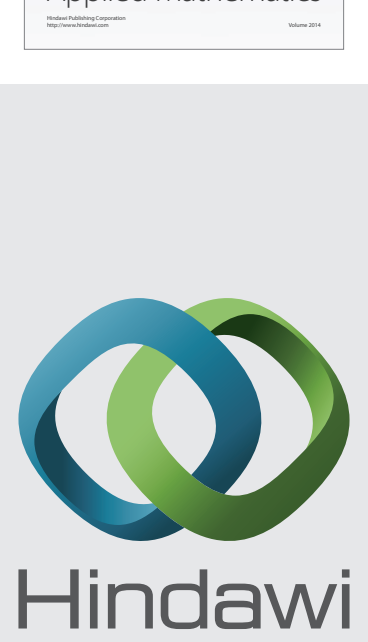

Submit your manuscripts at http://www.hindawi.com
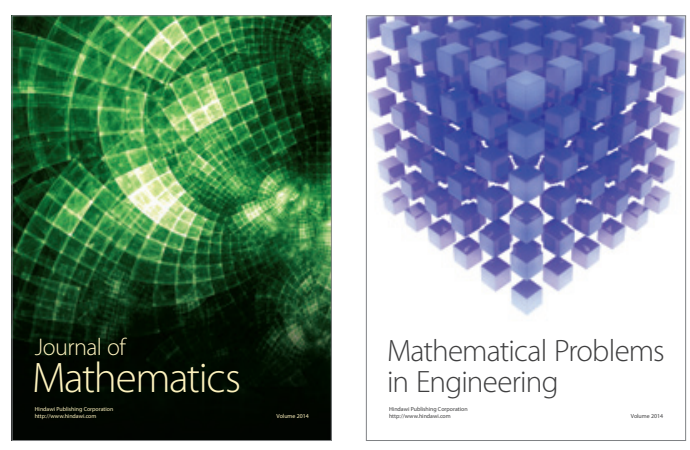

Mathematical Problems in Engineering
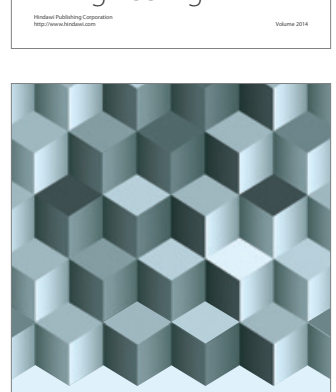

Journal of

Function Spaces
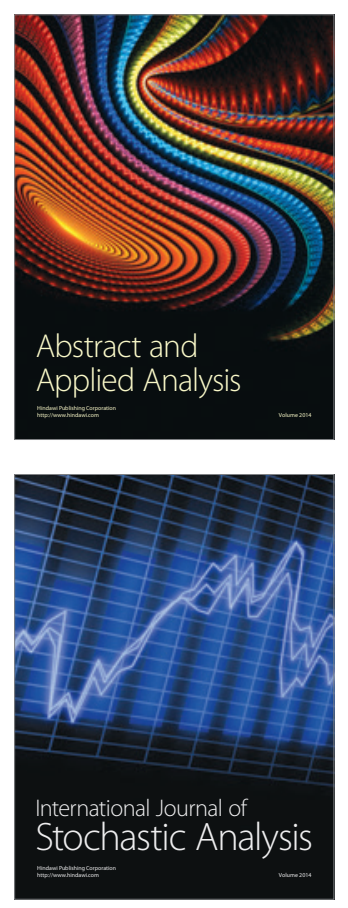

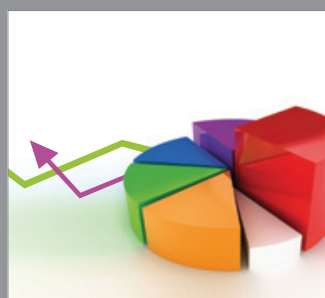

ournal of

Probability and Statistics

Promensencen
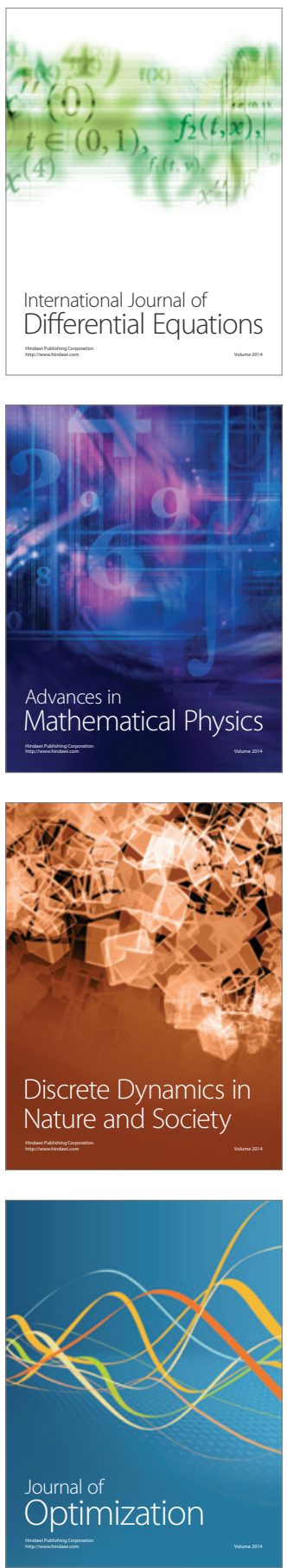